\title{
Converting habits of antibiotic use for respiratory tract infections in German primary care - study protocol of the
} cluster-randomized controlled CHANGE-3 trial

Anja Wollny ${ }^{1}$, Attila Altiner ${ }^{1}$, Tonia Brand ${ }^{4}$, Katharina Garbe ${ }^{1 *}$ (D), Martina Kamradt ${ }^{5}$, Petra Kaufmann-Kolle ${ }^{4}$, Mirko Leyh ${ }^{3}$, Regina Poß-Doering ${ }^{5}$, Joachim Szecsenyi ${ }^{5}$, Lorenz Uhlmann², Arwed Voss ${ }^{3}$, Dorothea Weber ${ }^{2}$, Michel Wensing ${ }^{5}$ and Christin Löffler ${ }^{1}$

\begin{abstract}
Background: The overuse of antibiotics is a major cause for the worldwide rise of antibiotic resistance. Although it is well known that acute respiratory tract infections (ARTI) are mainly caused by viruses and are often self limiting, antibiotics are too frequently prescribed in primary care. CHANGE-3 examines whether a complex intervention focusing on improving communication and provision of prescribing feedback reduces antibiotic use in patients suffering from ARTI.

Methods/design: The CHANGE-3 trial is a cluster-randomized controlled trial nested within a web-based public campaign conducted in two regions in Germany. A total of 114 medical practices will be included. Practices randomized to the intervention will receive a practice-specific antibiotic-prescription feedback and an educational outreach visit. During the visit the whole practice team will receive an introduction to e-learning modules addressing patient-centered communication on antibiotics. Furthermore, the practices will receive tablet PCs with information on antibiotics and the treatment of ARTI to be presented to patients. Practices randomized to the control will provide care as usual. The primary outcome measure is the antibiotic prescribing rate for patients with a history of ARTI. Data collected before the intervention, during the intervention and after the intervention will be compared. The use of narrow- vs. broad-spectrum antibiotics will be analyzed as a secondary outcome. A process evaluation is also part of the trial.
\end{abstract}

Discussion: This study should contribute to the growing body of research on reducing antibiotic prescription. Trial registration: ISRCTN, ISRCTN15061174. Registered retrospectively on 13 July 2018.

Keywords: Antibacterial agents, Drug resistance, Drug prescriptions, Respiratory tract infections, Primary health care, Randomized controlled trial

\footnotetext{
* Correspondence: katharina.garbe@med.uni-rostock.de

${ }^{1}$ Institute of General Practice, Rostock University Medical Center, Rostock,

Germany

Full list of author information is available at the end of the article
}

(c) The Author(s). 2019 Open Access This article is distributed under the terms of the Creative Commons Attribution 4.0 International License (http://creativecommons.org/licenses/by/4.0/), which permits unrestricted use, distribution, and reproduction in any medium, provided you give appropriate credit to the original author(s) and the source, provide a link to the Creative Commons license, and indicate if changes were made. The Creative Commons Public Domain Dedication waiver (http://creativecommons.org/publicdomain/zero/1.0/) applies to the data made available in this article, unless otherwise stated. 


\section{Background}

The excessive use of antibiotics in human and veterinary medicine is responsible for the worldwide rise of antibiotic resistance $[1,2]$. In Germany, approximately $40-45 \%$ of all antibiotics prescribed in human medicine stem from primary care, with acute respiratory tract infections (ARTI) [3] being the most common cause. Although among both experts and non-professionals it is well known that ARTI are primarily caused by viruses and are mostly self-limiting, in too many cases antibiotics are prescribed [4-6]. The main reasons for the inadequate prescription of antibiotics in primary care include a misleading safety culture and general practitioners' (GPs) misconception that patients would expect an antibiotic prescription [7, 8]. This leads to over-prescribing of broad-spectrum antibiotics such as quinolones and cephalosporins [3]. This eventually leads to increasing rates of antibiotic resistance, avoidable side effects and drug interactions as well as unnecessary direct and indirect costs, e.g., for hospital admissions [9-13].

Since the 1990s, efforts to reduce unnecessary antibiotic prescriptions in primary care have been made $[3,14]$. Trials have proven that some interventions are able to lower medication prescription rates in a relevant manner $[15,16]$. However, the effect of interventions is varying and is specific to situations and conditions [17]. A recent systematic review on interventions aiming at reducing antibiotic prescription for ARTI patients showed, for instance, positive effects of single-element and multifaceted interventions using GP communication skills' training and point-of-care testing. Other elements, such as patient-centered information alone, had no effect [16]. Wensing et al. showed efficacy of quality circles and written feedback on professional performance and prescribing [18]. So far, most interventions focus on GPs $[15,19]$. Research has shown that sensitizing the practice staff for a better interaction with patients carries the potential to relieve the GP's work and to contribute to improved decision-making in general [20,21]. Practice staff can play an important role in transferring knowledge to patients [21]. Thus, the idea of addressing the whole practice team in interventions to reduce unnecessary antibiotic prescriptions is gaining momentum.

\section{Aim}

The cluster-randomized controlled CHANGE-3 trial (cRCT) aims at testing the effect of a practice intervention including practice-specific antibiotic-prescription feed back, an education outreach visit, patient-centered communication, and patient information on the rate of antibiotic prescription for ARTI in German primary care.

Patient-centered communication will be conveyed via e-learning modules. Versions for physicians and practice staff members will differ and will be targetgroup-specific.
For the delivery of patient information, practices will receive tablet PCs with multimedia patient information on antibiotics and the treatment of ARTI.

The cRCT is nested within a larger-scale, web-based, public campaign conducted in two German states and has the title "Weniger Antibiotika: mehr Ideen" (English; less antibiotics: more ideas) [22]. Whereas the campaign tests the effectiveness of informing the patient and, to some extent, health care provider information at large, the cRCT investigates whether this intensified approach produces further effects. GP practices of the control group will provide care as usual, but will have access to the web-based campaign just like the general public.

\section{Methods/design \\ Trial design}

CHANGE-3 is a two-arm cRCT with GP practices and their patients forming a cluster. It is nested within a web-based public campaign conducted in two German regions. The antibiotic prescription rates of participating GPs will be measured over two successive winter seasons (Fig. 1). Additionally, the quality of prescriptions in terms of active ingredient chosen (broad- vs. narrow-spectrum antibiotics), usage of quinolones, seasonal fluctuations, and the knowledge of patients about antibiotics and treatment of ARTI will be monitored. GP practices will be allocated randomly either to the intervention group (IG) or the control group (CG). In the latter, care will be provided as usual. Claims data from the largest German statutory health insurance provider, AOK, will be used for all analyses. The SPIRIT checklist for this protocol can be found in Additional file 1 .

\section{Recruitment}

GP practices will be recruited in the two German states, Mecklenburg-Western Pomerania (MV) and BadenWurttemberg (BW). A total of 114 practices will be included in the trial (38 GPs in MV and 76 GPs in BW). GPs will be approached via invitation letters. Interested physicians will receive trial information via telephone or during a practice visit. Participating GPs will provide informed consent in writing. They will also agree to release their patients' anonymized prescription data as stored by the German statutory health insurance provider AOK $(\$ \$ 295,300$ SGB V). These data include all AOK-insured patients diagnosed with ARTI who receive medical treatment during the trial. Using these data, overall and practice-specific antibiotic prescription rates will be determined.

In addition, two written patient surveys will be conducted over two successive winter seasons. In each survey participating GP practices will recruit up to 50 ARTI patients aged 17 years or over to fill in a questionnaire. The questionnaire will measure patients' knowledge of 


\begin{tabular}{|c|c|c|c|c|c|}
\hline & \multicolumn{5}{|c|}{ STUDY PERIOD } \\
\hline \multirow[b]{2}{*}{ TIMEPOINT } & \multirow[t]{2}{*}{ Enrolment } & \multirow{2}{*}{$\begin{array}{c}\text { Allocation } \\
\text { T0 }\end{array}$} & \multicolumn{2}{|c|}{$\begin{array}{c}\text { Post- } \\
\text { allocation }\end{array}$} & \multirow[t]{2}{*}{ Close-out } \\
\hline & & & T1 & T2 & \\
\hline \multicolumn{6}{|l|}{ ENROLMENT: } \\
\hline Eligibility screen & $X$ & & & & \\
\hline \multirow{2}{*}{$\begin{array}{l}\text { Informed consent } \\
\text { Random Allocation }\end{array}$} & $\mathrm{X}$ & & & & \\
\hline & & $\mathrm{X}$ & & & \\
\hline \multicolumn{6}{|l|}{ INTERVENTIONS: } \\
\hline $\begin{array}{r}\text { prescription- } \\
\text { feedback }\end{array}$ & & & $X$ & $X$ & \\
\hline $\begin{array}{r}\text { educational } \\
\text { outreach visit }\end{array}$ & & & $X$ & & \\
\hline e-learning & & & - & & \\
\hline \multicolumn{6}{|l|}{ website / tablets } \\
\hline \multicolumn{6}{|l|}{ flyers } \\
\hline \multicolumn{6}{|l|}{ ASSESSMENTS: } \\
\hline $\begin{array}{r}\text { antibiotic } \\
\text { prescription rate }\end{array}$ & $X$ & & $X$ & $X$ & $X$ \\
\hline
\end{tabular}

Fig. 1 Overview of enrollment, intervention, and assessments of the CHANGE-3 trial

antibiotics as well as expectations and experiences related to antibiotic prescribing. Patients' participation in the survey will be anonymous and not related to any affiliation with a specific health insurance provider. Exclusion criteria are: not being able to read or fill in the questionnaire.

\section{Intervention}

The intervention is multifaceted and includes different elements (Additional file 2). The IG will receive feedback on practice-specific antibiotic prescription on two occasions (Additional file 2, component 1) and an educational outreach visit (Additional file 2, component 2), which is a central focus of the intervention. During the visit, a specially trained expert will discuss the practicespecific antibiotic-prescription feedback provided in detail. Additionally, the practice will have access to e-learning modules focusing on communication strategies, e.g., on how to handle patients expecting the prescription of antibiotics (Additional file 2, component 3 and 4). Patient information on the common cold, middle ear infections, and sinus infections is tailored to different groups of patients, e.g., the elderly or parents of children with ARTI, and is provided in the form of flyers and information on tablet computers (Additional file 2, components 5 and 6). Communication designers will be involved in the development of the materials.

\section{Control}

Patients of the CG will receive care as usual. Since the cRCT will take place during the web-based public campaign, GP practices of the CG will have access to the campaign's materials as the general public does.

\section{Data collection, completeness and quality}

Recruitment of GPs started in August 2017 and will continue until September 2018. The anonymized prescribing data from the GPs' patients will be retrieved at the 
beginning of the trial (baseline, T0) and over two successive winter seasons: during the intensified intervention phase in the first winter season (T1) and the second winter season (T2). That means that baseline data will be retrieved retrospectively after provision of written informed consent by GPs. The intervention will start after baseline collection is completed and continue throughout the trial, due to the public campaign running in parallel. To identify participating GPs in the secondary health data from AOK, two GP identifiers will be collected ("Lebenslange Arztnummer"; LANR and "Betriebsstättennummer"; BSNR). In addition, the following information will be gathered at the start of or during the trial: age and gender of GP, time since practice setup, specialist title of GP, and practice characteristics, like number of team members and field of expertise.

Also, up to 50 patient questionnaires on patient knowledge of antibiotics and treatment of ARTI will be delivered by the GPs. Participation of ARTI patients is voluntary and anonymous. This will be done throughout the entire trial. The questionnaires will collect information on patients' age and gender, employment situation, first language, educational background, knowledge on antibiotics, and reason for consultation. To avoid duplicates, participants who fill out the questionnaire will be listed anonymously (date of questionnaire dissemination, year of birth, and gender).

\section{Randomization}

Participating GP practices will be randomly assigned in a 1:1 ratio into the two study groups after recruitment. Randomization will be stratified by rate of antibiotic prescription for ARTI at baseline per practice. Randomization will be performed by the Institute of Medical Biometry and Informatics at the University Hospital Heidelberg.

\section{Outcome measures}

The primary outcome measure is the GPs' antibiotic prescription rate for ARTI at the end of the trial in comparison with the baseline data. These outcome data come from routinely collected data that are provided by the German statutory health insurance provider AOK. Tables 1 and 2 summarize relevant Anatomical Therapeutic Chemical (ATC) and International Classification of Diseases (ICD) codes, respectively, for calculating antibiotic prescription rates.

The secondary outcome is the choice of active ingredient (broad- vs. narrow-spectrum) in the antibiotic prescribed.

Additionally, data for the outcomes 'patients' level of knowledge on antibiotics' and 'patients' expectations and experiences related to antibiotic prescribing' are collected via questionnaires in two written patient surveys.

Table 1 Relevant Anatomical Therapeutic Chemical (ATC) codes

\begin{tabular}{|c|c|c|c|}
\hline Drug & ATC code & Drug group & Group name \\
\hline \multirow[t]{17}{*}{$\begin{array}{l}\text { Definition of classified ATC } \\
\text { codes }\end{array}$} & $\begin{array}{l}\text { J01FA (without } \\
\text { J01FA01) }\end{array}$ & $\begin{array}{l}\text { Macrolides (without erythromycin) - broad-spectrum } \\
\text { antibiotics }\end{array}$ & Macrolides \\
\hline & J01DB & First-generation cephalosporins & Cephalosporins \\
\hline & J01 DC & Second-generation cephalosporins & Cephalosporins \\
\hline & J01DD & Third-generation cephalosporins & Cephalosporins \\
\hline & J01DE & Fourth-generation cephalosporins & Cephalosporins \\
\hline & J01MA & Fluoroquinolones & Gyrase inhibitors \\
\hline & $\mathrm{J} 01 \mathrm{CR}$ & $\begin{array}{l}\text { Penicillins including beta-lactam inhibitors - broad-spectrum } \\
\text { antibiotics }\end{array}$ & Penicillins + beta-lactam-inhibitors \\
\hline & J01CA & Penicillins with extended spectrum & $\begin{array}{l}\text { Penicillins with extended } \\
\text { spectrum }\end{array}$ \\
\hline & J01 CE & Beta-lactamase-sensitive penicillins & Beta-lactamase-sensitive penicillins \\
\hline & J01EE01 & Sulfamethoxazole and trimethoprim & $\begin{array}{l}\text { Sulfamethoxazole and } \\
\text { trimethoprim }\end{array}$ \\
\hline & J01XE01 & Nitrofurantoin & $\begin{array}{l}\text { Trimethoprim, nitrofurantoin, } \\
\text { fosfomycin }\end{array}$ \\
\hline & J01EA01 & Trimethoprim & $\begin{array}{l}\text { Trimethoprim, nitrofurantoin, } \\
\text { fosfomycin }\end{array}$ \\
\hline & J01XX01 & Fosfomycin & $\begin{array}{l}\text { Trimethoprim, nitrofurantoin, } \\
\text { fosfomycin }\end{array}$ \\
\hline & J01FA01 & Erythromycin & Erythromycin \\
\hline & J01FA16 & Solithromycin & Erythromycin \\
\hline & J01A & Tetracyclines & Tetracyclines \\
\hline & J01other & J01-other & Other antibiotics \\
\hline
\end{tabular}


Table 2 Relevant International Classification of Diseases, version 10 (ICD-10) codes

\begin{tabular}{llll}
\hline Diagnoses & ICD-10 & Indication & ICD group \\
\hline Definition of classified & $J 20 ; J 21.0 ; J 21.1 ; J 21.8 ; J 21.9 ; J 22 ; J 40$ & Acute bronchitis & Lower respiratory infection \\
ICD-10 codes & $J 00 ; J 02.0 ; J 02.8 ; J 02.9 ; J 04 ; J 06 ; J 10.1 ; J 11.1$ & Acute infection of the upper respiratory tract & Upper respiratory infection \\
& $J 01$ & Acute sinusitis & Upper respiratory infection \\
& J03.0; J03.8; J03.9 & Tonsillitis & Upper respiratory infection \\
& H65.0; H65.1; H65.9; H66.0; H66.4; H66.9 & Otitis media & Upper respiratory infection
\end{tabular}

The surveys' results are meant as a feedback to the practices about their patients' perspective. The results of the second patient survey will show, in comparison with the first ones, whether the project interventions resulted in changes concerning patients' expectations and patients' knowledge. Descriptive statistics and specific tests for group comparison will be used to analyze survey data (IBM SPSS statistics 20).

\section{Methods to reduce bias and enhance data quality}

With reference to the Cochrane Collaboration's tool for assessing risk of bias [23], a number of procedures are carried out to reduce potential sources of bias. Firstly, to reduce selection bias, participating GP practices in both states are recruited with identical procedures. A non-responder analysis will also be performed. Secondly, randomization of participating practices will be performed electronically by statisticians not involved in physician recruitment. Thirdly, specified inclusion and exclusion criteria (ICD and ATC codes) will be used to calculate antibiotic prescription rates for ARTI among participating practices in order to reduce the risk for information bias and selective outcome reporting. Fourthly, to reduce variation in intervention delivery, outreach visits will be standardized. Due to financial and time constraints within this trial it will not be possible to blind participants and trial staff. Prior to its start, the study was registered in the public trial archive ISRCTN.

\section{Sample size calculation}

We expect to find a relative reduction of the antibiotic prescription rate of $30 \%$ (which means an absolute reduction from 40 to $28 \%$ ) between the groups. The sample size considerations are based on the chi-squared test using a significance level of $\alpha=5 \%$ (two-sided) and a power of $(1-\beta)=80 \%$. This results in a sample size of $n=244$ patients per group. To take the clustered structure into account, we assumed an intra-cluster correlation coefficient (ICC) for patients in practices of 0.2 [24]. This value is based on previous studies. The cluster size is assumed to be $m=80$ patients. Thus, the design effect $(D E)$ equals $D E=1+(m-1) \times I C C=1+79 \times 0.2=$ 16.8. These considerations result in a sample size of $N=n$ $\times D E=244 \times 16.8=4100$ patients per group. Thus, the total sample size is 104 practices of size $m=80$ resulting in
8320 patients. A drop-out rate of about $8 \%$ is expected and, therefore, 10 additional practices will be recruited (see Fig. 2 for detailed information on the scheduled flow of patients). The analysis will be done by using a logistic mixed-effects model, which we expect to explain more of the variance in the prescription of antibiotics. Therefore, the model should be more powerful than the chi-squared test. The calculations are done using SAS 9.4 (Proc Power).

\section{Statistical analyses}

In a first step, descriptive statistics will be provided for the primary and secondary outcomes as well as for patient, practice, and GP characteristics, including mean, standard deviation, median, interquartile range (IQR), minimum and maximum for continuous variables, and frequency in percentages for categorical variables. The primary objective of this study is to examine the difference in antibiotic prescription rate after intervention (T1) between the two study arms.

The confirmatory analysis of the primary endpoint will be conducted based on the intention-to-treat (ITT) population, where all patients will be included in the analysis and assigned to the group they were randomized to.

A logistic mixed-effects model will be applied to assess the respective hypothesis of equal rates in both groups regarding the primary endpoint using a significance level of $\alpha=5 \%$. A random intercept will be included for practices. Furthermore, age and gender of patients will be included as covariates. Missing values regarding the primary endpoint will be imputed using multiple imputation. As a sensitivity analysis, the primary endpoint will be evaluated on the per-protocol population, where patients with major protocol violations will be excluded. As a further sensitivity analysis, the baseline prescription rate on practice level will be included as an additional covariate in the primary logistic mixed-effects model.

Mixed models, as described for the primary endpoint, will be used to analyze the secondary outcomes. All calculated $p$ values regarding secondary endpoints will be of a descriptive nature. A detailed statistical analysis plan will be written prior to the final analysis. SAS (SAS Institute Inc., Cary, NC, USA) version 9.4 or higher will be used to carry out the analyses. A non-responder analysis is planned regarding size of practice, number of antibiotic prescriptions in case of ARTI, and application of broad-spectrum antibiotics. 


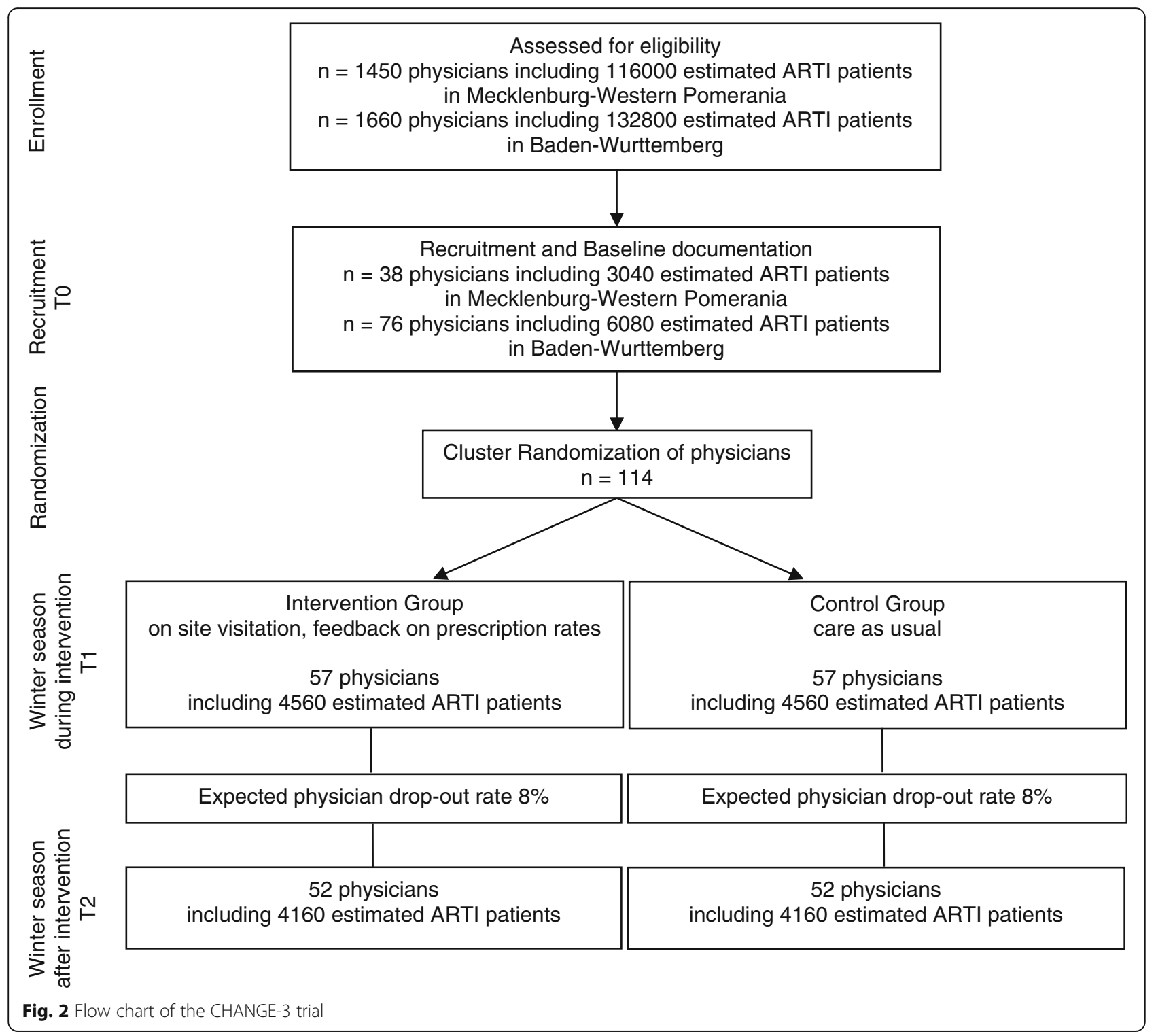

\section{Process evaluation}

To understand the mechanisms of the educational components, a process evaluation is accompanying the intervention in the CHANGE-3 study. Using a mix of qualitative and quantitative research methods, the process evaluation aims at assessing reach and fidelity of the implementation program, its effects on daily practice of health care delivery to ARTI patients and the impact of diverse context factors on health services. The survey as well as the interview guide used within the process evaluation are based on the Theoretical Domains Framework (TDF) $[25,26]$ to gain insight into working mechanisms. The survey focuses on aspects regarding the regional intervention with multimedia content (web-based public campaign), the practice team intervention with outreach visit, e-learning and feedback report and on personal perceptions regarding the provision of health services to ARTI patients. Interviews will add in-depth understanding of educational intervention components, context factors impacting on the uptake of the intervention and the significance and role of the implemented set of measures to practice teams and patients.

In the IG, data collection will occur through telephone and face-to-face interviews with GPs, medical assistants (GP practice nurses), patients, and outreach visitors as well as surveys for health care providers. In the CG, patient interviews and provider surveys will be used to collect data. Survey data will be collected twice. All physicians and medical assistants in the IG and CG will be invited to participate in the process evaluation. A 
purposive sampling strategy will be followed to recruit interview participants among GPs and medical assistants $(n=20)$, patients $(n=20)$, and outreach visitors $(n=5)$.

Interviews will be recorded and transcribed. The pre-defined categories of a framework, such as Flottorp et al. [27] or Atkins et al. [26], will be used to identify determinants of practice which influence health care delivery to ARTI patients in general practices, with regard to the educational components of the intervention and with regard to converting habits of antibiotic use. Descriptive statistics as well as specific tests for group comparison will be used to analyze survey data. Further analyses which investigate the relationship between survey-based and claims-data-based outcomes will be applied if the sample size is sufficiently large. All collected data will be pseudonymized for analysis.

The results of the process evaluation will strengthen the reporting in CHANGE-3 and provide information for future implementation programs.

\section{Discussion}

As a cRCT, CHANGE-3 aims at improving antibiotic prescription among ARTI patients cared for in general practice. The cRCT is nested in a large public campaign that is geared towards sensitizing the population of two German states for the reasonable use of antibiotics.

CHANGE-3 is based on two previous studies (CHANGE and CHANGE-2) investigating the efficiency of improved physician-patient communication, patient empowerment and point-of-care testing on antibiotic prescription rates $[19,24]$. The study benefits from lessons learnt from these previous trials and, beyond that, includes recent findings on the importance of patient information, on a practice-based approach and on the effect of practice-specific prescription feedback [28, 29]. CHANGE-3 is designed to test the hypothesis that a relative reduction of $30 \%$ in antibiotic prescription can be achieved by comparatively simple means.

\section{Trial status}

Recruitment of GPs will be finished in September 2018. Recruitment of patients is ongoing throughout the trial.

\section{Trial registration}

The trial is registered at ISRCTN under the reference ISRCTN15061174. Registered retrospectively on 13 July 2018; http://www.isrctn.com/ISRCTN15061174.

\section{Additional files}

Additional file 1: SPIRIT checklist: recommended items to address in a clinical trial protocol. (DOCX $64 \mathrm{~kb})$

Additional file 2: Intervention description according to Template for Intervention Description and Replication (TIDieR) [30]. (DOCX 96 kb)

\section{Abbreviations}

ARTI: Acute respiratory tract infections; BSNR: "Betriebsstättennummer" is a unique number which identifies every practice in Germany; BW: BadenWurttemberg; CG: Control group; DE: Design effect; GP: General practitioner; ICC: Intra-cluster correlation coefficient; IG: Intervention group; IQR: Interquartile range; ITT: Intention-to-treat; LANR: "Lebenslange Arztnummer" is a unique identification number for each physician in Germany; MV: Mecklenburg-Western Pomerania

\section{Acknowledgements}

Not applicable.

\section{Funding}

CHANGE-3 is funded by the German Ministry of Health (Bundesministerium für Gesundheit (BMG)) under the reference ZMVI1-2516FSB100. The sponsor has no role in the design of the study or the writing of the manuscript, nor do they have a role in the collection, analysis, or interpretation of the data.

Availability of data and materials

Not applicable.

\section{Authors' contributions}

AW drafted the manuscript. AA designed the study. TB participated in coordination. KG drafted the manuscript. MK provided expertise on process evaluation. PKK designed the study. ML was involved in the development of the intervention materials. RPD provided expertise on process evaluation. JS designed the study. LU provided statistical expertise. AV was involved in the development of the intervention materials. DW provided statistical expertise. MW designed the study. CL drafted the manuscript. All authors critically read and approved the final manuscript.

\section{Ethics approval and consent to participate}

The trial was approved by the Ethics Committee at the Rostock University Medical Center before recruitment of physicians and patients, 4 September 2017, ref. A 2017-0134. The process evaluation was approved by the Ethics Committee at the Medical Faculty, Heidelberg, 11 June 2018, ref. S-349/2018.

Consent for publication

Not applicable.

Competing interests

The authors declare that they have no competing interests.

\section{Publisher's Note}

Springer Nature remains neutral with regard to jurisdictional claims in published maps and institutional affiliations.

\section{Author details}

${ }^{1}$ Institute of General Practice, Rostock University Medical Center, Rostock, Germany. ${ }^{2}$ Department of Medical Biometry, Institute of Medical Biometry and Informatics, University Hospital Heidelberg, Heidelberg, Germany. ${ }^{3}$ Department of Communication Design and Media, University of Applied Sciences, Technology, Business and Design, Wismar, Germany. ${ }^{4}$ aQua-Institute for Applied Quality Improvement and Research in Health Care GmbH, Göttingen, Germany. ${ }^{5}$ Department of General Practice and Health Services Research, University Hospital Heidelberg, Heidelberg, Germany.

Received: 13 November 2018 Accepted: 17 January 2019

Published online: 06 February 2019

References

1. Bundesministerium für Gesundheit. DART 2020. 2015. https://www.bmel.de/ SharedDocs/Downloads/Broschueren/DART2020.pdf?_blob=publicationFile. Accessed 18 July 2018

2. Roca I, Akova M, Baquero F, Carlet J, Cavaleri M, Coenen S, et al. The global threat of antimicrobial resistance: science for intervention. New Microbes New Infect. 2015:6:22-9.

3. Bundesamt für Verbraucherschutz und Lebensmittelsicherheit. Germap 2015 2017. https:/www.bvl.bund.de/SharedDocs/Downloads/05 Tierarzneimittel/ germap2015.pdf?_blob=publicationFile\&v=9. Accessed 18 July 2018. 
4. KBV. Rationale Antibiotikatherapie bei Infektionen der oberen Atemwege. In: Wirkstoff AKTUELL. 2012. https://www.akdae.de/Arzneimitteltherapie/WA/ Archiv/Antibiotika-URTI.pdf. Accessed 18 July 2018.

5. KBV. Rationale Antibiotikatherapie bei Infektionen der unteren Atemwege In: Wirkstoff AKTUELL. 2017. https://www.akdae.de/Arzneimitteltherapie/WA/ Archiv/Antibiotika-LRTI.pdf. Accessed 18 July 2018.

6. Altiner A, Bell J, Duerden M, Essack S, Kozlov R, Noonan L, et al. More action, less resistance: report of the 2014 Summit of the Global Respiratory Infection Partnership. Int J Pharm Pract. 2015;23(5):370-7.

7. Kumar S, Little P, Britten N. Why do general practitioners prescribe antibiotics for sore throat? Grounded theory interview study. BMJ. 2003; 326(7381):138

8. Petursson P. GPs' reasons for "non-pharmacological" prescribing of antibiotics. A phenomenological study. Scand J Prim Health Care. 2005; 23(2):120-5.

9. Höffken G, Lorenz J, Kern W, Welte T, Bauer T, Dalhoff K, et al. Epidemiology, diagnosis, antimicrobial therapy and management of community-acquired pneumonia and lower respiratory tract infections in adults. Guidelines of the Paul-Ehrlich-Society for Chemotherapy, the German Respiratory Society, the German Society for Infectiology and the Competence Network CAPNETZ Germany. Pneumologie. 2009;63(10):e1-68.

10. Schröder H. Hände weg von der eisernen Reserve. Gesundheit und Gesellschaft. 2012;7-8/11:21-7.

11. Del Mar CB, Glasziou PP, Spinks AB. Antibiotics for sore throat. Cochrane Database Syst Rev. 2006;(4):CD000023.

12. German College of General Practitioners and Family Physicians (DEGAM). Halsschmerzen DEGAM-Leitlinie Nr. 14. 2009. https:/www.degam.de/files/ Inhalte/Leitlinien-Inhalte/Dokumente/DEGAM-S3-Leitlinien/Leitlinien-Entwuerfe/ 053-010_Halsschmerzen/LL-14_Langfassung_ZD.pdf. Accessed 23 Mar 2018.

13. National Institute for Health and Clinical Excellence. Respiratory tract infections - antibiotic prescribing. NICE clinical guideline 69. 2008. https:// www.nice.org.uk/guidance/cg69/evidence/full-guideline-196853293. Accessed 23 Mar 2018.

14. Bundesinstitut für Arzneimittel und Medizinprodukte, Paul-Ehrlich-Institut. Bulletin zur Arzneimittelsicherheit - Informationen aus BfArM und PEl. 2015. https://www.bfarm.de/SharedDocs/Downloads/DE/Arzneimittel/ Pharmakovigilanz/Bulletin/2015/3-2015.pdf?_blob=publicationFile\&v=6 Accessed 23 Mar 2018.

15. Coxeter P, Del Mar CB, McGregor L, Beller EM, Hoffmann TC. Interventions to facilitate shared decision making to address antibiotic use for acute respiratory infections in primary care. Cochrane Database Syst Rev. 2015;(11): CD010907.

16. Köchling A, Löffler C, Reinsch S, Hornung A, Böhmer F, Altiner A, Chenot J-F. Reduction of antibiotic prescriptions for acute respiratory tract infections in primary care: a systematic review. Implement Sci. 2018;13(1):47.

17. Arnold SR, Straus SE. Interventions to improve antibiotic prescribing practices in ambulatory care. Cochrane Database Syst Rev. 2005;(4): CD003539.

18. Wensing M, Broge B, Riens B, Kaufmann-Kolle P, Akkermans R, Grol R, et al Quality circles to improve prescribing of primary care physicians. Three comparative studies. Pharmacoepidemiol Drug Saf. 2009;18(9):763-9.

19. Altiner A, Berner R, Diener A, Feldmeier G, Köchling A, Löffler C, et al. Converting habits of antibiotic prescribing for respiratory tract infections in German primary care-the cluster-randomized controlled CHANGE-2 trial. BMC Fam Pract. 2012;13:124.

20. Freund T, Peters-Klimm F, Boyd CM, Mahler C, Gensichen J, Erler A, et al. Medical assistant-based care management for high-risk patients in small primary care practices: a cluster randomized clinical trial. Ann Intern Med. 2016;164(5):323-30.

21. Chapman SA, Blash LK. New Roles for Medical Assistants in Innovative Primary Care Practices. Health Serv Res. 2017;52:383-406.

22. Weniger Antibiotika: mehr Ideen; 2018. https://www.weniger-antibiotika.de. Accessed 30 October 2018

23. Higgins JPT, Green S (editors). Cochrane handbook for systematic reviews of interventions. John Wiley and Sons Ltd, The Atrium, Southern Gate, Chichester, West Sussex PO19 8SQ, England; 2008.

24. Altiner A, Brockmann S, Sielk M, Wilm S, Wegscheider K, Abholz HH. Reducing antibiotic prescriptions for acute cough by motivating GPs to change their attitudes to communication and empowering patients: a cluster-randomized intervention study. J Antimicrob Chemother. 2007;60(3): 638-44.
25. Francis JJ, O'Connor D, Curran J. Theories of behaviour change synthesised into a set of theoretical groupings: introducing a thematic series on the theoretical domains framework. Implement Sci. 2012;7:35.

26. Atkins L, Francis J, Islam R, O'Connor D, Patey A, Ivers N, et al. A guide to using the Theoretical Domains Framework of behaviour change to investigate implementation problems. Implement Sci. 2017;12(1):77.

27. Flottorp SA, Oxman AD, Krause J, Musila NR, Wensing M, Godycki-Cwirko M, et al. A checklist for identifying determinants of practice: a systematic review and synthesis of frameworks and taxonomies of factors that prevent or enable improvements in healthcare professional practice. Implement Sci. 2013;8:35

28. Hallsworth M, Chadborn T, Sallis A, Sanders M, Berry D, Greaves F, et al. Provision of social norm feedback to high prescribers of antibiotics in general practice: a pragmatic national randomised controlled trial. Lancet. 2016;387:1743-52

29. Meeker D, Linder JA, Fox CR, Friedberg MW, Persell SD, Goldstein NJ, et al. Effect of behavioral interventions on inappropriate antibiotic prescribing among primary care practices: a randomized clinical trial. JAMA. 2016;315(6): 562-70.

30. Hoffmann TC, Glasziou PP, Boutron I, Milne R, Perera R, Moher D, et al. Better reporting of interventions: template for intervention description and replication (TIDieR) checklist and guide. BMJ. 2014;348:g1687.

\section{Ready to submit your research? Choose BMC and benefit from:}

- fast, convenient online submission

- thorough peer review by experienced researchers in your field

- rapid publication on acceptance

- support for research data, including large and complex data types

- gold Open Access which fosters wider collaboration and increased citations

- maximum visibility for your research: over $100 \mathrm{M}$ website views per year

At BMC, research is always in progress.

Learn more biomedcentral.com/submissions 